CAHIERS DE

NARRATOLOGIE

\section{Cahiers de Narratologie}

Analyse et théorie narratives

15 | 2008

Récits et genres historiques

\title{
Temps, récit et vérité historique chez Antonio de Herrera y Tordesillas
}

\section{Renaud Malavialle}

\section{(2) OpenEdition}

Journals

Édition électronique

URL : http://journals.openedition.org/narratologie/698

DOI : 10.4000/narratologie.698

ISSN : 1765-307X

Éditeur

LIRCES

Référence électronique

Renaud Malavialle, «Temps, récit et vérité historique chez Antonio de Herrera y Tordesillas », Cahiers de Narratologie [En ligne], 15 | 2008, mis en ligne le 14 décembre 2008, consulté le 22 avril 2019. URL http://journals.openedition.org/narratologie/698; DOI : 10.4000/narratologie.698

Ce document a été généré automatiquement le 22 avril 2019

\section{(c) (i) (9)}

Cahiers de Narratologie - Analyse et théorie narratives est mis à disposition selon les termes de la licence Creative Commons Attribution - Pas d'Utilisation Commerciale - Pas de Modification 4.0 International. 


\title{
Temps, récit et vérité historique chez Antonio de Herrera y Tordesillas
}

\author{
Renaud Malavialle
}

1 L'œuvre d'Antonio de Herrera y Tordesillas (1549-1625) offre l'exemple d'une pensée historique novatrice dans la Monarchie Catholique du début du XVII siècle. Il a contribué à fonder un champ d'activité en quête de règles autonomes de fonctionnement. L'étude des écrits théoriques du chroniqueur des Indes permet de souligner ses conceptions du temps de l'histoire et des rapports de l'écriture historienne avec le pouvoir. Outre son intégration des acquis de la critique historique de son époque, nous analyserons sa position face à un cas difficile de falsification afin de prendre la mesure des limites de l'école historienne hispanique au tournant des XVI ${ }^{\mathrm{e}}$ et XVII ${ }^{\mathrm{e}}$ siècles.

Conception de l'histoire et récit des origines

2 L'historiographie d'Antonio de Herrera marque l'accomplissement et un premier dépassement du courant humaniste.

3 Il est le premier historien officiel (Cronista Mayor) des Indes Occidentales ${ }^{1}$ et sa grande synthèse de la conquête et de la colonisation du «Nouveau Monde» est plus qu'une chronique ou un témoignage vécu. Cette œuvre confirme son auteur par la masse et la variété de sa documentation. Son expérience du monde politique, militaire, administratif et diplomatique acquise auprès de ses protecteurs de haut rang l'a conduit à la pratique d'une histoire contemporaine, en prise directe avec les affaires politiques de la Monarchie Catholique. De nombreuses approbations et censures rédigées au titre de sa fonction de chroniqueur du roi l'ont averti des méthodes, des contraintes et des écueils propres à l'histoire ancienne. Il a par ailleurs contribué à l'édition de textes de la tradition littéraire médiévale qui présente des rapports étroits avec les problématiques de l'histoire gothique. L'approbation au Romancero general y flor de diversa poesía de Miguel de Madrigal $^{2}$ est éditée en 1605. C'est toutefois sur ses écrits théoriques que nous concentrerons ici notre analyse. 
4 Sa carrière commence en 1570 au service de Vespaciano Gonzaga Colonna, vice-roi de Navarre. Il acquiert alors son expérience administrative et politique avant de le suivre en Italie. Dès la mort de Gonzaga en 1586, Herrera sert la Couronne et jouit d'une confortable autonomie financière. En 1593, il traduit à la demande du roi le tacitiste italien Giovanni Botero $^{3}$ avant de cumuler les charges de Cronista Mayor de Indias (1596) et celle de Cronista de Castilla dès 1598.

5 En 1601, il s'installe à Valladolid où il travaille à l'édition de la Historia general de los hechos de los castellanos en las islas i tierra firme del mar océano, rédigeant ses Discursos ${ }^{4}$. Une édition imprimée en 1804 en a repris la plupart ${ }^{5}$. Avec trois manuscrits inédits que nous avons reproduits, ils offrent un regard cohérent sur la théorie herrerienne de l'histoire ${ }^{6}$, posthumaniste et tacitiste ${ }^{7}$.

6 Les Discursos sont dédiés à de grands personnages de la monarchie, du duc d'Albe au Compte-Duc d'Olivares ${ }^{8}$. Conformément à son époque, l'écriture de l'histoire a une fonction édifiante. Mais elle l'est désormais sans évacuer l'épaisseur temporelle. Elle rompt avec le dialogue sans intermédiaire entre antiquité et temps modernes ${ }^{9}$. La conception pragmatique qui triomphe au tournant des $\mathrm{XVI}^{\mathrm{e}}$ et $\mathrm{du} \mathrm{XVII}^{\mathrm{e}}$ siècle est issue d'une tension logique entre deux tendances. La première répond à l'exigence de spécification dans l'analyse d'une situation historique. La seconde, héritière de l'humanisme cicéronien, machiavélien ou tacitiste, soumet l'intérêt de l'histoire aux enseignements que l'on peut extraire des événements politiques par généralisation, par l'élaboration inductive de normes censées régler la conduite politique.

7 Ces deux tendances sont contradictoires mais absolument complémentaires. La tradition annalistique vouée à préserver de l'oubli les faits et gestes des Princes complète la théorie politique moderne formulée dans et par le récit historique.

8 Dans le sillon de la tradition italienne, la formulation cicéronienne s'est imposée. Or le principe de l'historia magistra vitae s'inscrivait dans un horizon intellectuel gréco-latin spécifique. Les historiens et penseurs politiques italiens (Machiavel, Guichardin) redécouvrent en Hérodote, Thucydides et Polybe, Tite-Live ou Tacite un savoir politique naturel où les méthodes de gouvernement en temps de paix comme en temps de guerre fournissent des exemples stratégiques applicables à toute époque et en tous lieux. Herrera le commente à son tour :

Cicerón [...] llama a la historia maestra de la vida y luz de la verdad, porque sucediendo siempre unos mismos accidentes, como son mudanzas de reinos y estados, los pasados nos instruyen para que determinando sobre lo presente conozcamos lo venidero. ${ }^{10}$

9 L'enregistrement des res gestce acquiert une utilité politique dès lors que, comme le proclame Cabrera de Córdoba dans son De historia, paraentenderla y escribirla : " una misma manera de mundo es todo ». Autrement dit, si les événements du passé et ceux du présent sont du même ordre, en vertu de règles connues ou formulables par induction, il est possible d'anticiper sur l'avenir dès lors que les circonstances présentes sont identifiées. La connaissance de l'histoire fonde la science politique, elle-même pensée sur le modèle épistémique du droit et de la médecine : elle doit conduire à la prudentia.

10 Cette conscience historique repose sur une conception circulaire du temps dont Platon a formulé un modèle, qui n'admet de changements de régime politique qu'en tant que dégradations et régénérations cycliques d'un régime politique idéal. 
11 Herrera ne retient en effet des propositions des maîtres de l'Antiquité que celles qui confirment le caractère normatif de la démarche historienne. Le temps historique demeure-t-il exclusivement cyclique chez Herrera?

$\mathrm{Au} \mathrm{XVI}^{\mathrm{e}}$ siècle, c'est avant tout à une méthode de lecture que les théoriciens de l'histoire réfléchissent. Leur attention se porte sur sa finalité générique conformément aux données sociales de l'émergence du genre: l'histoire s'écrit pour les Princes et les seigneurs, à leur commande et dans leur intérêt. La vertu ultime de ces lectures, si elles contribuent à la formation du Prince, c'est la démultiplication infinie du champ d'expérience qu'elles lui proposent :

Y porque son muchas y muy varias las cosas particulares que se han de conocer, no basta una sola experiencia, porque la vida del hombre es breve espacio para conseguir la perfección de esta virtud con solas las cosas que él mismo ve y trata, y por ésta podrá adquirir con dificultad esta ciencia de bien vivir tan necesaria en todo tiempo y a todos estados, pudiéndose este tal siendo mozo llamar viejo, porque son pocas las cosas que respecto a las sucedidas en tantos siglos se pueden ver en una sola edad. ${ }^{11}$

13 À la finitude de la vie humaine individuelle s'oppose l'infinitude du réel, à laquelle l'histoire ouvre accès. Or cette amplification de perspective implique un renversement du temps de l'histoire, une variante du renversement du temps observable chez tous les augustiniens à cet époque ${ }^{12}$, chez tous ceux qui célèbrent les progrès de l'humanité ${ }^{13}$ comme un organisme vivant ${ }^{14}$.

Face au temps destructeur, celui de l'oubli de l'expérience passée, face au temps immobile ou stagnant de la tradition morale, voici un temps nouveau, facteur de l'idée de progrès. Grâce à l'expérience de ses prédécesseurs intégrée précocement, le prince prudent pourra faire preuve de maturité malgré son jeune âge («pudiéndose este tal siendo mozo llamar viejo »). La prudence repère des relations régulières de cause à effet et ne compare les situations à travers le temps (« todo lo sucedido en los tiempos pasados y comparándolo con lo presente ») que pour repérer les conditions d'actions égales ("y conociendo en qué parte corresponde a sus obras, y en qué son diferentes de ello»). Avant la célèbre variation de Pascal ${ }^{15}$ sur le motif des «nains assis sur les épaules de géants » ${ }^{16}$, Herrera rend compte de la temporalité scientifique émergeante. Le point de vue est seulement inversé : Pascal dépeint la communauté des hommes sous les traits d'un seul individu accumulant le savoir ; Herrera souligne qu'un lecteur peut intégrer toutes les expériences humaines dignes de mémoire ${ }^{17}$.

15 Dans les deux cas, le temps devient le lieu de l'édification d'une vérité pratique, il cesse déjà d'être le «temps de la petite histoire anecdotique, répertoire d'accidents vides de sens et vaine surcharge pour la mémoire, mais un temps réduit à la raison et mis au service de la vérité $»^{18}$. La rencontre des principes cycliques de l'historia magistra vitae et de la dynamique progressive de la temporalité augustinienne conduit de la répétition du Même à la dialectique du semblable évolutif ou progressif. Le Prince conscient des lois de l'histoire peut tenter de s'en affranchir. Bel exemple de subversion moderne de l'esprit augustinien: la dynamique de la théologie de l'histoire est mise au service d'un engagement confiant dans la réalisation temporelle d'une histoire intramondaine ${ }^{19}$.

Le temps formateur des épreuves collectives

Herrera présente la Reconquête comme une période d'épreuves formatrice d'un caractère politique. La tradition épique a préparé la grande historiographie qu'il voit éclore, remplissant le rôle d'une histoire peu diffusée et que l'effort de guerre oblige à négliger. Il 
justifie ainsi les lacunes de l'historiographie espagnole contre le constat que les grandes nations sont celles qui connaissent leur passé. Les Espagnols durent « faire primer l'action sur le discours » : la proposition est inversée et suggère un degré d'activité guerrière, noble s'il en est, au-delà duquel l'écriture de l'histoire est un loisir nécessairement ajourné. Cette argumentation s'inscrit dans le contexte de la guerre idéologique des représentations des passés monarchiques.

verre affirme la maturité des temps et la place privilégiée de la Monarchie espagnole. Cette prépondérance n'a pas toujours prévalu, elle ne correspond pas à une position essentiellement dominante de l'Espagne ou à une supériorité innée. Les premiers habitants de la Péninsule sont en effet décrits selon un primitivisme ambigu: "En la primera edad vivían los hombres en España con simplicidad, sin contiendas y sin cuidado de muchas cosas. ${ }^{21}$ Pareille vision ne repose sur aucune documentation historique, juste sur une image traditionnelle de l'harmonie primordiale, de la paisible rusticité. Cette étape projette sur le plan politique celle de l'enfance chez l'individu et devient une condition de possibilité du progrès des arts et des mœurs :

En la segunda se pasó debajo del imperio de los Cartagineses, Romanos, Godos y otros, y habiéndose tomado experiencia y conocimiento de muchas cosas, especialmente con la violencia y astucias de los Moros y Arabes, se vino a conseguir una gran prudencia humana y militar. ${ }^{22}$

Grâce à de prestigieux précepteurs, les premiers Espagnols vont apprendre les rudiments de la politique et de l'art militaire. Avant l'autonomie, le tutorat des Carthaginois et des Romains est une initiation précoce à la violence et au combat contre les infidèles. Elle admet une dialectique du recyclage du mal : la confrontation avec la violence et la rouerie (« violencia y astucias ») des Maures permet l'apprentissage de la vertu.

La période d'occupation musulmane est donc un âge de formation. Les plus méritants obtiennent des formes de préséance politique et symbolique et parmi les peuples hispaniques, les Castillans sont le fer de lance :

La tercera edad fue hasta que los Españoles, y particularmente los castellanos con sus propias fuerzas, sin ayuda ni consejo de otra ninguna nación ni Príncipe, echaron de su tierra a estos Mahometanos, en que se gastaron muchos años, en los cuales, por la religión y por la patria pelearon hechos maravillosos, cuales en tanto discurso de tiempo no se hallan continuados en ninguna parte del mundo. ${ }^{23}$

Les exploits des Castillans se distinguent par leur grandeur mais surtout par une durée formatrice. La durée historique de la période musulmane et de son influence sur la formation d'une identité chrétienne exclusive et fortement ancrée territorialement («por la religión y por la patria. $\left.»^{24}\right)$ est assumée. Le contraste est saisissant avec Cabrera de Córdoba qui la dévaluait. Chez Herrera elle est singularisante et l'épaisseur du passé patriotique est ainsi soulignée. En toile de fond apparaissent la signification du cours des temps, la lutte pour la reconquête catholique, pour la patrie, le dénominateur commun de tous les Espagnols fédérés autour des héroïques Castillans, dignes avant-gardes qui donnent du sens à l'histoire. Vue par Cabrera comme une étape scandaleuse, une éclipse sans consistance historique, la présence musulmane est chez Herrera une mise à l'épreuve formatrice d'une personnalité singulière. Qu'aucune nation au monde n'ait pris le relais d'une telle geste atteste que les Espagnols sont le nouveau peuple élu ; mais cette élection apparait comme le fruit naturel d'une histoire en construction et non comme une essence éternelle ou prédestinée.

L'Espagne accomplie 
21 Une quatrième période s'ouvre lorsqu'une intégrité territoriale est retrouvée. La reconquête achevée de la patrie permet l'accès à la plénitude de la souveraineté :

La quarta edad comenzó desde que echado de España el mahometismo, fue esta nación gloriosamente dilatando su Imperio en Europa, en Africa, y en las regiones occidentales y orientales con trabajos, quales ninguna otra pudiera sufrir ni continuar con tanta constancia ${ }^{25}$.

Le passage d'une position défensive à une attitude offensive traduit une continuité distinctive d'un tempérament formé au cours des siècles, privilège de l'Espagne. Ce dernier âge est enfin celui de l'expérience exportée, de la mission civilisatrice selon la même légitimité que celle reconnue aux Romains dans le Discurso y tratado que la felicísima Monarquía castellana fue acrecentando su Imperio por los mismos modos que la República Romana :

La Ciudad de Roma [...] volvió a la autoridad Consular, con que sujetando a sus vecinos y sojuzgada Italia, sacó fuera sus fuerzas ampliando su Imperio; y la Monarquía castellana [...] en echando a los Mahometanos de España, pasó sus armas en Italia y otras partes. ${ }^{26}$

Dans le Discurso XII, Herrera pousse à l'extrême le parallélisme historique entre Rome et la Castille: la conquête de territoires italiens avant celle de nouvelles terres montre une Castille reprenant légitimement le flambeau impérial, longtemps tenu par Rome : «Con que ha llegado la felicísima grandeza que ahora tiene $\aleph^{27}$. L'héritière de Rome est le noyau de l'Empire hispanique à vocation universelle. Les Castillans sont l'avant-garde de la chrétienté dont la Monarchie catholique assure la défense. Dans cette authentique théorie du temps de l'histoire hispanique peut se déployer un projet d'écriture : ainsi se trouvent surmontés l'annulation du temps propre aux humanistes (retour aux sources gréco-latines et mépris pour la période gothique) et temps continu et progressif propre aux interprétations chrétiennes depuis la théologie de l'histoire.

Ethos historiens

Antonio de Herrera s'est posé la question des critères de la vérité historique. C'est surtout l'auteur lui-même qui est sujet à caution. La quantité d'information qu'il a accumulée et la difficulté que représente toute tentative de vérification obligent à examiner la crédibilité morale de la "cause efficiente». Même si les conditions d'exercice du métier sont désormais interrogées, c'est avant tout les indices de partialité qui seront pourchassés dans le discours.

Les théoriciens reprennent la réflexion des Anciens sur l'ethos de l'historien. Juan Luis Vives les y avait fortement engagés : elle tenait le plus souvent lieu de méthode. Evaluer l'indépendance du jugement, l'absence relative de pression sont les priorités. Citons Herrera :

Y sobre todo tenga el ánimo libre : este tal hará bien el oficio de historiador [...], lo cual no podrá hacer si teme, o tiene esperanza de premio, o tiene odio contra alguno. $^{28}$

Antonio Domínguez Ortíz évoque la censure exigée par des individus ou des groupes lésés dans leur réputation ${ }^{29}$. José Godoy Alcántara ${ }^{30}$ cite le cas du conseiller d'État Juan de Velasco qui jugeait certains historiens "trop curieux" pour avoir mis en doute la prédication de l'apôtre saint Jacques en Espagne ${ }^{31}$. Les protections de Cabrera de Córdoba ne lui évitèrent pas l'exil et ne permirent pas la publication de ses Relaciones. L'écriture des événements d'Aragon de 1591 impliquait les droits constitutionnels du souverain face aux lois fondamentales du royaume; ce cas de pression institutionnelle est emblématique 32. 
Herrera en a bien entendu connu de semblables. J.- F. Lázaro a montré qu'il a tenu à maintenir son critère historiographique ${ }^{33}$ face à Francisco Arias Dávila y Bobadilla, comte de Puñonrostro, qui contestait le traitement de son aïeul dans la Historia general de los hechos de los castellanos. Le comte eut accès au manuscrit d'Herrera et fit pression sur le Conseil des Indes ${ }^{34}$ pour interdire la publication et si le conseil donna raison à l'auteur ${ }^{35}$, Herrera n'évoque pas en vain le besoin de protection. "Igual Juez y tan apacible con todos, no sujeto al señorío ni voluntad de nadie, sin imaginar ni pensar en qué ha de dar gusto $»^{36}$. Telle est l'utopie de Herrera : n'avoir pas même idée des pressions potentielles, dans la ligne de Pedro de Navarra ${ }^{37}$. Telle est la revendication d'autonomie du jugement de la sphère savante. La vérité documentée doit primer sur toute autre considération. Une telle conception requiert un espace relativement stable, une distance respectable entre la production du discours officiel et les intérêts politiques immédiats. Lui correspond une stratégie de promotion et d'affectation du désintéressement visant à rendre la critique plus difficile. Il s'agit de penser un intérêt collectif à l'échelle de l'État moderne et de ses prétentions à se situer au-dessus des partis.

Champ professionnel

Herrera appartient à la génération immédiatement postérieure au grand historien aragonais Jerónimo Zurita et à son émule castillan Ambrosio de Morales, deux des maîtres de la critique historique et précurseurs du champ historien ${ }^{38}$. La charge de chroniqueur du roi, dont il est titulaire, n'existe que depuis un demi-siècle environ et il a conscience des moyens très limités dont l'historien dispose, des obstacles propres au manque de tradition. La difficulté spécifique du métier lui apparaît comme un argument de prestige de la discipline: "A la historia no incita ningún provecho sino la honra, por esto es ejercitada de emperadores y reyes y gente principal. $»^{39}$.

Cet argument fréquent selon lequel d'illustres auteurs ont anobli l'histoire est fréquent chez Cabrera de Córdoba. Mais l'argumentation de Herrera est beaucoup plus subtile et profonde. Elle dénote un haut niveau de conscience de l'intérêt symbolique que la profession peut espérer de «l'affectation du désintéressement $»^{40}$. L'historien de métier négocie de cette façon son entrée dans une forme d'aristocratie du savoir au sein d'une république protégée.

L'appel au mécénat d'Etat illustre cette stratégie discursive : Herrera aussi évoque les dépenses engagées par Alexandre le Grand en faveur de l'histoire, citant même Budé qui en a fait le compte.

31 Dans ce passage historiens mercenaires, poètes épiques et savants désintéressés se côtoient. L'histoire offre bien des exemples de l'importance du mécénat des Princes :

Los Cretenses dieron una cantidad de oro por las obras de Homero. Nicomedes Rey de Chipre dio a Isocrates por el libro que le dedicó 120 ducados. Los Romanos levantaron una estatua a Josefo por la historia que hizo de Captivitate judaica. Y nuestro Rey Alonso el octavo hizo a Don Rodrigo Ximenez de Rada, primado de las Españas y Arzobispo de Toledo. ${ }^{41}$

32 Le projet d'une histoire nationale s'inscrit dans la ligne de l'historiographie alphonsine, depuis le De rebus Hispaniae Liber de Rodrigo Jiménez de Rada et la Chrónica general supervisée par Alphonse X de Castille ${ }^{42}$. Encourager l'écriture et la publication des ouvrages d'historiens, selon d'illustres modèles, c'est pour le Prince faire preuve de conscience et de compétence politiques. Le geste d'Herrera est plus qu'un signe, il est aussi un acte positif qui contribue à une forme d'accumulation première d'un capital scientifique : 
No tuviéramos las que hay, si no fuera por la grandeza de ánimo y afición que tuvo a

las buenas letras el Pontífice León Décimo, que las sacó de poder de bárbaros. ${ }^{43}$

L'idée de la destruction du savoir fait dramatiquement apparaitre le bénéfice collectif de son élaboration et de sa conservation. Herrera esquisse ainsi une sociologie de la science, les conditions minimales de l'institution scientifique. L'histoire révèle le lien intime qui unit l'État et la science dans un projet collectif de conservation souveraine des monarchies. Nous en retrouvons une très semblable illustration en France dans les Additions à l'histoire de Louys $\mathrm{XI}^{44}$ de Gabriel Naudé qui insistait aussi sur l'importance de l'histoire comme connaissance et sur le rôle que le progrès des lettres joue dans l'accroissement des empires ${ }^{45}$. Chez Herrera aussi, cette revendication de dignité fonde les «linéaments d'une historiographie critique », selon l'heureuse formulation de YvesCharles Zarka.

es principes et les conditions sociales d'une première forme d'objectivité professionnelle s'affirment par la fondation d'une sociologie politique du savoir où c'est encore l'histoire qui fournit les exemples à méditer.

Antonio de Herrera y Tordesillas et les faussaires

Dans le Discurso y tratado que Beroso, Metastenes, Manetón y un breviario de los tiempos que tiene nombre de Filón son falsos e inventados por Juan Annio de Viterbo, inédit, on peut observer les connaissances de Herrera des travaux de ses prédécesseurs pour assainir la bibliographie. Ce discours de circonstance est destiné à prévenir son destinataire contre les fausses chroniques, il a cependant une valeur scientifique générale :

Acordándome de haber visto entre los libros de vd unas artes de lo que escrivió Juan Annio de Viterbo con el nombre de Beroso, envío a vd lo que después de mucho estudio he hallado en graves autores para que vd conozca el engaño que de él han recibido hombres muy señalados, por que si fuere posible no incurran otros en los mismos yerros ${ }^{46}$

Dans une visée collective et solidaire, d'illustres naïfs sont dénoncés comme Jean Nauclerus et Jean Driedon que Cabrera de Córdoba avait invoqués comme des autorités déduisant des Commentaria du moine de Viterbe des éclairages chronologiques. Herrera juge ainsi Annius de Viterbe :

[...] Hizo muy grandes yerros en la coronografía [...] de la cual depende la certidumbre de la historia de donde sucedió que personas muy graves y católicas, sin echar de ver el engaño, cayeron en él y estos fueron Juan Driedón, Nauclero, Valerio Anselmo... ${ }^{47}$

Herrera a lu les réfutations érudites ou tout au moins la synthèse de Melchior Cano ${ }^{48}$. Le théologien reconnaît à son tour ses dettes envers Juan de Vergara qui avait réfuté Annius de Viterbe, dans le dialogue $\mathrm{V}$ des Ocho cuestiones del Templo ${ }^{49}$ :

Muchos son los autores que han apurado esta verdad y la han sacado a luz, y en particular fray Melchor Cano en el libro 11 de sus lugares comunes teológicos, el Volterrano en el libro 14 de su Antropología, Juan de Bergara en sus ocho questiones, Luis Vives en el libro 5 del modo de enseñar las disciplinas [.... $]^{50}$

La méthode d'Annius de Viterbe est démasquée : l'usage indiscriminé ou habilement panaché de documents authentiques et de faux entièrement fabriqués à partir d'extrapolations bibliographiques et grâce à de véritables compétences d'érudit. La démystification est un acquis savant («es cosa muy evidente») à proclamer pour qu'historiens et curieux gagnent un temps précieux. La première erreur d'Annius de Viterbe est son excessive confiance, sa façon de sous-estimer son prochain : 
El verdadero Beroso escrivió su historia hasta Ciro, el que vivió más de 600 años después de la guerra de Troya y este Beroso de Juan Annio reduce las cosas hechas por los Asirios y Caldeos solamente hasta el principio del reino troyano que viene ser hasta el 18 Rey de los Asirios que fue contemporáneo de Danao, primero rey de Troya. ${ }^{51}$

Il faudrait non seulement ignorer l'œuvre de Bérose mais encore négliger l'information de Flavius Josèphe dont le Contra Appianus rappelle l'étendue du champ historique couvert par le prélat babylonien :

Demás de esto compárese por Josepho en lo que escrivió Contra Apión: eran tres libros de el verdadero Beroso que trató de las cosas de los caldeos y de Juan Annio no parece más de que uno que habla de las cosas de los Asirios. ${ }^{52}$

Annius de Viterbe dissimule des sources, en invente d'autres et finit par se contredire :

El mismo Annio contradice a su Metastenes por que dice que Beroso escrivió la historia de los reyes de Asiria hasta Sardanapalo, último Rey de aquella monarquía, mentira clara y conocida..$^{53}$

Herrera invoque l'extrême invraisemblance d'une vérité solitaire sur des thèmes pareils. Il faut plus de cohérence pour ne pas éveiller les soupçons de la communauté savante. Il rapporte en outre les divergences fondamentales sur la succession des rois perses et la durée de leurs règnes que Juan de Vergara avait exposées méthodiquement avant d'envisager les plus probables. Vergara, lui, proposait au lecteur un examen rationnel, minutieux et vérifiable. Au prix d'un long et rigoureux effort de concentration, il est en effet possible à tout curieux de suivre son raisonnement :

Es cosa cierta y notoria que desde Ciro hasta Alexandro Magno hubo 14 reyes de Persia y que duró 230 años y este Metastenes de Annio no muestra más de 8 reyes, y no quiere que el imperio de los Persianos haya durado mas de 190 años. ${ }^{54}$

Nous ne suivrons pas Herrera dans les détails de son compte rendu de la réfutation où Juan de Vergara démontre qu'Annius de Viterbe a commis des erreurs sur chacun des auteurs qu'il a falsifiés. Signalons seulement la présence du prêtre égyptien Manéthon et de Philon:

El $3^{\circ}$ autor en quien también Annio anduvo ciego es Manetón [que] escribió de las cosas de los Egipcios muy diferente de lo que trató el verdadero [...] el $4^{\circ}$ es Filón de quien dice que escribió un breviario de los tiempos. ${ }^{55}$

Craignant d'être fastidieux, le résumé devient schématisation inintelligible pour qui n'a pas lu Juan de Vergara. L'humaniste évoquait l'agacement de Tite-Live déplorant régulièrement dans son histoire $a b$ urbe condita de ne pas disposer de sources sûres pour la Rome archaïque. Prétendre que Philon avait prolongé l'œuvre de l'historien romain en utilisant comme lui des documents officiels était particulièrement maladroit. Ce qui demeurait possible pour l'œuvre d'un auteur hébreu mal connu était bien grossier dans le cas de Tite-Live.

4 Enfin la comparaison stylistique formulée par Juan de Vergara entre l'élégance du Philon attesté et la trivialité des textes composés par le faussaire ne pouvait abuser : « no tienen cosas que parezcan de este Filón de Annio, porque son excelentes en el estilo, eloquencia, gravedad, fidelidad y en la verdad, nada de lo cual se halla en este Filón. ${ }^{56}$.

Herrera fait donc preuve d'une connaissance appréciable du plus lourd dossier de critique historique du XVI ${ }^{\mathrm{e}}$ siècle. Sa synthèse de l'acquis érudit traduit une réelle connaissance des mécanismes de l'imposture qui contraste avec l'inconscience ou la légèreté de Cabrera de Córdoba.

Les limites de la critique face aux récits des faussaires 

pas toujours parvenu à l'illustrer à son tour. Il a rédigé un compte rendu de l'affaire des récits apocryphes et des reliques de la Torre Turpiana et du Sacromonte de Grenade ${ }^{57}$, où la distance fait défaut et où de nombreux groupes de pouvoirs sont concernés. Le Resumen de lo que pasó en el descubrimiento de las santas reliquias de la ciudad de Granada ${ }^{58}$ reprend les conclusions de Gregorio López Madera ${ }^{59}$. De 1588 (reliques de la Torre Turpiana) à 1595 (livres de plomb du Sacromonte), une série de textes et de documents, rouleaux de plomb gravés de textes arabes, restes humains et autres reliques, tisse un récit accréditant la venue de Saint Jacques en Espagne, le martyre de ses disciples à Grenade même. Certains textes présentaient par ailleurs la domination maure comme un vecteur d'unité de la foi en un syncrétisme original destiné à réconcilier les confessions en conflit sur le sol ibérique. José Godoy Alcántara attribue ces impostures à Alonso del Castillo et Miguel de Luna, deux morisques chargés eux-mêmes d'examiner les documents. Traducteur de l'arabe de Philippe II, Alonso del Castillo avait une rare maitrise non seulement du grec et du latin, mais de l'arabe dialectal et de celui des érudits andalous.

Les premières lignes du manuscrit de Herrera rappellent la date du commencement de l'affaire et donnent le ton : « El año de 1588 felicísimo por este caso [...] derribándose en la ciudad de Granada una torre antigua [...] se halló una caja de plomo, betunada por de dentro y por de fuera. $»^{60}$. Le chroniqueur par ailleurs si sensible aux vertus de la raison s'émeut d'emblée ici d'une manifestation surnaturelle. L'arcana dei recèle une prophétie de saint Jean, l'apôtre au style prophétique et eschatologique le plus vigoureux :

Una relación en latín de Patricio sacerdote, que dice que la escritura es una profecía de san Juan evangelista y que el lienzo es la mitad del paño en que nuestra señora limpió sus lágrimas en la pasión de su bendito Hijo. ${ }^{61}$

Évangélisme, messianisme et mariologie, la spiritualité hispanique et populaire est habillement sollicitée par les faussaires. En 1595 apparaissent les reliques annoncées en une miraculeuse et providentielle confirmation: "Una masa de ceniza y huesos quemados y una calavera, dientes y muelas, todo con mucho olor y fragancia. $»^{62}$

Leligiosité hispanique n'a pas pu rompre le cercle vertueux (ou vicieux) et magique des confirmations réciproques entre récits et documents reliquaires. Le génie diabolique des faussaires offrait entre autres merveilles les secrets de la nature divine ( $«$ Liber de essencia $\left.D e i{ }^{63}\right)$ en caractères anciens et historicisés capables d'abuser, selon Herrera, les philologues avertis de la corruption ou de l'évolution des langues:

La letra de las dichas cuatro láminas y de las cubiertas de los libros dicen fundamentum ecclesia y essencia Dei y está escrito discretísimamente y como de persona que sabía cómo se había de escribir en plomo, para mayor perpetuidad. ${ }^{64}$

50 Herrera donne foi à la mise en œuvre naturelle des desseins de la Providence et il évoque les procédures d'authentification conduites par l'archevêque de Grenade, Pedro de Castro y Quiñones, qui a requis l'intervention de doctes experts pour obtenir une datation approximative :

Los peritos en sus artes depusieron y declararon que son antiguos de más de mil años sin fingimiento ni artificio y también dijeron los árabes Doctos que fueron traídos de diversas parte que eran antiquísima la gramática. ${ }^{65}$

51 Conformément à une procédure scientifique rigoureuse qui fait appel aux meilleurs experts, prend soin de choisir leur provenance afin d'éviter les jugements intéressés ou impliqués, les découvertes sont authentifiées : 
La gramática, los frasis, y modos de hablar y que algunas dictiones y las letras sin puntos, aunque los puntos diferenciában la potestas de las letras en la lengua árabe y que no era posible ser compuestos por Árabes modernos y que si muchos Árabes se juntaran para hacerlo, de cien años acá no lo supieran hacer. ${ }^{66}$ documents apocryphes ont su conjurer. Par rapport aux affabulations d'Annius de Viterbe, le rapport de force avec la critique apparaît ici inversé. Cette fois ce sont les critiques qui ne conçoivent pas la prudence, l'ingéniosité philologique et la maîtrise des «sciences annexes » de l'histoire que les faussaires ont mises en œuvre. La motivation de la Cause morisque a été largement sous-estimée: la capacité d'un peuple blessé à fabriquer les pièces les plus convaincantes pour sa justification. Le branle-bas institutionnel pour la qualification des précieux documents suit son cours sous l'égide du roi :

Para proceder en negocio tan grave con el tiento y autoridad conveniente [...] el Arzobispo señaló día para proceder a la calificación de las reliquias y avisó al Nuncio. ${ }^{67}$

démarche conciliaire à l'échelle locale puis "nationale » se met en place. Une culture, une langue et des préoccupations communes présidées sous la juridiction de la monarchie confessionnelle sont concernées :

Ya sus cabildos y otros Principales Prelados de Castilla y del Andalucía y a los Provinciales Abades y Prelados de todas las órdenes Monásticas y Mendicantes, a los Catedráticos de las universidades y Ministros del Santo Officio de la Inquisición [...].

Comme l'usage des majuscules le souligne, la procédure implique toutes les autorités et les institutions morales, spirituelles, judiciaires et savantes convoquées par l'autorité suprême de la Monarchie. Tout le corps du royaume de Castille est concerné par la délibération dans la ville andalouse. L'affaire locale des livres de plomb de Grenade acquiert ainsi les dimensions d'une authentique affaire d'État:

[...] Suplicándolos y pidiéndolos que autorizasen este acto con sus personas como la gravedad del negocio pedía para que más rigurosamente se examinase conforme a los Breves de su santidad y voluntad del Rey nuestro señor y de su consejo y público en todo el arzobispado de Granada [...].69

Mais l'ampleur du phénomène déborde le plan institutionnel: la dévotion populaire contribue à l'événement. Processions, prières publiques et messes enflamment l'atmosphère sociale et spirituelle de la ville andalouse.

Herrera insiste fortement sur les compétences réunies le jour choisi par l'archevêque ("muchos theologos»). Un vocabulaire charismatique propre à l'histoire répète le caractère autorisé de la délibération ( « muy graves personas »). Il redit la prise en compte des savoirs techniques appropriés à la nature des documents («los intérpretes y artífices »), souligne les origines diverses des experts consultés (« se trujeron de muchas partes ») et multiplie le lexique de l'enquête («diligencias »; « examen »). Il pondère le sérieux de l'entreprise en évoquant les délais convenables et adaptés dont ont bénéficié les savants pour rendre leur jugement critique ( muchos días, todo lo que fue necesario ») et fait enfin valoir un argument magistral qui évoque implicitement le miracle légendaire des traductions convergentes des auteurs de la Septante : « Y de una misma conformidad, determinaron y resolvieron ser tales Reliquias el paño de nuestra señora, [...] y el hueso de San Estebán. $»^{70}$ 

l'histoire péninsulaire. Sur quoi le Concile de Trente et une commission spécialement mandatée par le pape déclarent les reliques authentiques et véritables en ordonnant qu'elles soient vénérées. le plus réfléchi. Un effet de réel élaboré par des faussaires, aussi bien que par des historiens, conformément aux critères de vérité et aux codes sociaux et culturels admis à une époque est peut-être une imposture imparable. Parce qu'il se nourrit de la croyance religieuse, idéologique et même scientifique, l'effet de réel sait flatter toutes les mythologies et aucune époque ne saurait se protéger de ses formes abusives, tant l'imposture grandit toujours au sein de la certitude.

59 La difficulté d'Herrera à adopter une position critique s'explique par l'ampleur sans précédent d'un phénomène " de société ", peut-être aussi par sa disposition à admettre la nouveauté, certainement par l'avance durable des faussaires sur la critique. CEuvres de militants passionnés qui rédigent un document syncrétique d'une culture humiliée et surtout écartée de l'institution, les documents apocryphes ont fleuri sur la faiblesse des pouvoirs publics, lesquels s'étaient privés des compétences indispensables à un prompt déchiffrement de l'imposture philologique. La culture officielle, en renforçant les mécanismes d'exclusion et d'intransigeance religieuse, est ainsi magistralement prise à son propre piège, les compétences nécessaires pour déjouer la mystification lui font défaut. L'histoire n'aurait pas lieu d'être si la distance et l'effet décantatoire du temps ne pouvaient ainsi être savamment mis à profit.

60 L'éclectique chroniqueur officiel, si informé des avancées de la meilleure critique historique, a été parmi tant d'autres le jouet de l'imposture. Surévaluer le hiatus entre l'adhésion aux falsifications évangéliques et la synthèse critique que les Discursos mettent en lumière, serait une illusion rétrospective. Dans l'épaisseur du vécu de l'époque, le chroniqueur officiel est pris au piège de son appartenance à des instances dirigeantes et autorisées engagées dans l'authentification et la légitimation des documents apocryphes. L'historien pourtant conscient du montage d'Annius de Viterbe manque de distance et de sérénité dans une affaire d'une urgente actualité. Au regard de l'enthousiasme manifesté par Nicolás Antonio ${ }^{71}$, qui disposait pour sa part de décennies de recul, à la lecture des démonstrations de Gregorio López Madera, peut-on juger trop sévèrement pareille crédulité ?

61 Les Discursos d'Antonio de Herrera y Tordesillas expriment un moment fondateur. Le tournant des XVI et $\mathrm{XVII}^{\mathrm{e}}$ siècles voit les pratiques historiennes modernes théorisées, la critique historique reconnue, sinon établie sur des bases encore solides, et la pensée politique engagée dans le cours des temps. L'époque marque l'affirmation de l'histoire en tant que discipline. Elle devient théoriquement à la fois le fondement de la connaissance et l'aboutissement de tous les savoirs. À l'impérialisme épistémique correspond sur le plan de l'ethos de l'historien un vif désir de reconnaissance. Antonio de Herrera affirme le projet d'alliance historiographique du chroniqueur et de la monarchie : il prétend devenir le logos de la monarchie, le vecteur du discours souverain, la voix véridique de la parole officielle. Le sens du bilan bibliographique rigoureux distingue le chroniqueur des Indes Occidentales de Cabrera de Córdoba, qu'il dépasse sur un second plan : la conception du temps de l'histoire. Là où l'auteur du De historia ne voyait que barbarie et insignifiance, Antonio de Herrera y Tordesillas signale, en accord avec Juan de Mariana, un âge intermédiaire (médiéval, étymologiquement) qui a forgé une identité. Il restitue ainsi une

Cahiers de Narratologie, 15 | 2008 
épaisseur temporelle, il préserve la continuité des temps historiques d'une Espagne moins éternelle que progressivement constituée dans l'adversité. La société hispanique s'appuie sur la prédominance castillane dont la légitimité résulte du cours objectif des événements, fondateur d'une personnalité apte à fournir un socle national à vocation péninsulaire et universelle.

\section{NOTES}

1. Antonio de Herrera y Tordesillas, Historia general de los hechos de los castellanos en las islas y tierra firme del mar océano...en ocho década, desde 1492 o 1554, Madrid, 1601-1615, 4 vol.

2. Segunda parte del Romancero general y flor de diuersa poesia, recopilados por Miguel de Madrigal..., En Valladolid, Luis Sánchez, 1605.

3. Giovanni Botero, Diez libros de la Razón de Estado. Con tres libros las causas de la grandeza, y magnificiencia de las ciudades de Juan Botero. Traducido de Italiano en castellano, por mandato del Rey nuestro señor, por Antonio de Herrera su criado. En Madrid, Luys Sanchez. Año MDXCIII.

4. Primera parte de las varias Epístolas, Discursos y Tractados de Antonio de Herrera a diversos claros varones, las cuales contienen muchas materias utiles para el govierno político y milita. Con un elogio de la vida y hechos de el Lizdo. Xptoval Vaca de Castro del Consejo Supremo y Gobernador de los Reynos del Pirú, BNM, Mss., nº 3011.

5. Discursos morales, políticos e históricos inéditos de Don Antonio de Herrera y Tordesillas, cronista del Rey Don Felipe Segundo, autor de las Décadas de Indias, y de otras muchas obras, par Juan Antonio de Zamácola, Madrid, Imprenta de Ruiz,1804. 6. Herrera, op. cit., « Discurso y tratado sobre la materia de Estado », (fol. 50) ; « Discurso $\mathrm{y}$ tratado que Beroso, Metastenes, Manethon y un breviario de los tiempos que tiene nombre de Filon son falsos e inventados por Juan Annio de Viterbo »; « Resumen de lo que pasó en el descubrimiento de las santas reliquias de la ciudad de Granada, conforme a lo que escribió el doctor Gregorio López Madera, del Consejo Supremo de su Majestad, y otros autores ».

7. Selon les termes de José María Iñurrítegui Rodríguez.

8. Les dédicataires ne sont pas explicitement signalés. Dans l'article cité (p. 136) Iñurrítegui Rodríguez attribue la dédicace du « Discurso y Tratado que el medio de la historia es suficiente para adquirir la prudencia » $\left(\mathrm{n}^{\circ} 20\right)$ à Olivares et estime qu'il a été rédigé entre 1598 et 1605 .

9. Par « temps modernes ", nous entendons l'époque contemporaine de nos auteurs, qu'ils commencent à nommer par ces termes.

10.Antonio de Herrera y Tordesillas, Discursos morales, históricos y políticos, p.1 :

"Cicéron [...] appelle l'histoire maitresse de vie et lumière de vérité parce que comme les événements qui surviennent sont toujours du même ordre, qu'il s'agisse de changements de règne ou de gouvernement, ceux du passé nous enseignent à réfléchir sur le présent pour connaître l'avenir ». 
11. Herrera, op. cit., p. 23 : «Et parce qu'il y a beaucoup à connaître et en variété infinie, une seule expérience ne peut suffire ; la vie d'un homme est en effet bien brève pour atteindre la perfection de cette vertu, à l'aide seulement de ce qu'il voit ou de ce dont il s'occupe ; avec cette seule expérience, il aura du mal à acquérir cette science du bien vivre si nécessaire en tous temps et dans toutes les républiques; celui qui y parvient peut se dire âgé (sage) quoiqu'il soit jeune ; au regard de ce qui s'est passé en tant de siècle, il y a en effet peu de choses qui puissent être vécues en si peu de temps ".

12. Cf. Gérard Ferreyrolles, L'influence de la conception augustinienne de l'histoire au $\mathrm{XVII}^{\mathrm{e}}$ siècle, in : XVII ${ }^{\mathrm{e}}$ Siècle, $\mathrm{n}^{\circ} 135$, avril-juin $1982,34^{\mathrm{e}}$ année, $\mathrm{n}^{\circ} 2$.

13. Étienne Gilson, Introduction à l'étude de St Augustin, Vrin, $2^{\mathrm{e}}$ édition, 1943, p. 230. Voir aussi Les métamorphoses de la Cité de Dieu, Louvain, 1952.

14.Cf. Cité de Dieu, $X, 14$ et Francis Bacon : «C'est à la vieillesse du monde et à son âge mûr qu'il faut attacher ce nom d'antiquité. »

15. Après avoir constaté que l'homme «n'est produit que pour l'infinité », Pascal insiste sur le caractère cumulatif du savoir : "Par une prérogative particulière, non seulement chacun des hommes s'advance de jour en jour dans les sciences, mais que tous les hommes ensemble y font un continuel progrez à mesure que l'univers vieillit, parce que la mesme chose arrive dans la succession des hommes que dans les âges différents d'un particulier. De sorte que toute la suite des hommes, pendant le cours de tant de siècles doit être considérée comme un mesme homme qui subsiste toujours et qui apprend continuellement. » (Blaise Pascal, Fragment de préface pour le Traité du vide (1647) in CEuvres, Paris, Hachette, 1908, t. II, pp. 138 et 139).

16. Cette phrase souvent attribuée à Pascal est empruntée à Bernard de Chartres (XII ${ }^{\mathrm{e}}$ siècle) : « Nous sommes des nains assis sur des épaules de géants. Si nous voyons plus de choses et plus lointaines qu'eux, ce n'est pas à cause de la perspicacité de notre vue, ni de notre grandeur, c'est parce que nous sommes élevés par eux. ».

17. L'un des philosophes ou savants les plus souvent cités pour illustrer cette tendance est Francis Bacon : « La Vérité [est] fille du Temps et non de l'Autorité », Novum Organum, 1620 livre I, aph. 84 (p. 144 de la traduction française de M. Malherbe et J.-M. Pousseur, Paris, PUF, 1986).

18. Georges Gusdorf, « L'homme des Lumières », in Ilona Kóvacs (éd.), Les Lumières en Hongrie, en Europe centrale et en Europe orientale, Budapest, Akadémiai Kiadó, et Paris, Edition du CNRS, 1984, p. 34. Cité par Pierre André Taguieff, dans Du progrès, Essai. Librio 428, Paris, 2001.

19. Voir Etienne Gilson et aujourd'hui Marcel Gauchet.

20. Nous n'utilisons pas le terme de « nation » dans le sens que lui donnera le $\mathrm{XIX}^{\mathrm{e}}$ siècle européen. A une époque où la paroisse est un horizon culturel suffisant pour une proportion importante de la population, le sentiment religieux conditionne davantage les comportements que l'idée d'une appartenance à une communauté culturelle et territoriale partageant une histoire. Sur l'idée de « nation » à cette époque, voir Jean-Paul Le Flem, «L'idée de nation en Espagne au XVII ${ }^{\mathrm{e}}$ siècle », XVII ${ }^{\mathrm{e}}$ siècle, $\mathrm{n}^{\circ} 176$, Paris, Société d'Etude du XVII siècle, 1992 ; Jean-Frédéric Schaub, « L'histoire politique sans l'État : mutations et reformulations ", in Historia a debate, Tomo III, 1995, pp. 217-235 ; I. A. A. Thompson, «Castille, Spain and the monarchy : the political community from patria natural to patria national », in : Richarg L. Kagan and Geoffrey Parker (ed.), Spain, Europe and the Atalntic World, Cambridge, Cambridge University Press, 1995.

21.Herrera, op. cit., p. 37 : « Dans le premier âge, les hommes vivaient en Espagne dans la simplicité, sans se battre et insouciants pour l'essentiel ». 
22.Ibidem : « Le deuxième âge marque le passage sous l'empire des Carthaginois, des Romains, des Wisigoths et d'autres, et l'acquisition de l'expérience et des connaissances dans bien des domaines. En particulier, la violence et l'ingéniosité des Maures et des Arabes fut à l'origine d'une grande prudence humaine et militaire ».

23.Ibidem, p. 37 : « Le troisième âge dura jusqu'à ce que les Espagnols, et en particulier les Castillans par leurs propres moyens, sans l'aide et sans le conseil d'aucune autre nation ni d'aucun prince étranger, chassèrent les Mahométans de leur terre, ce qui exigea des années, pendant lesquelles, pour la religion et pour la patrie, il ont réalisé des exploits merveilleux, et renouvelés sur une telle durée qu'on n'en trouve d'exemple nulle part ailleurs dans le monde ».

24.Ibidem : " pour la religion et pour la patrie ».

25. Ibidem, pp. 37-38 : Le quatrième âge a commencé quand le mahométisme a été chassé d'Espagne ; cette nation a alors glorieusement augmenté son Empire en Europe, en Afrique, et dans les régions occidentales et orientales par des efforts qu'aucune autre n'aurait pu consentir et prolonger avec autant de constance ».

26. Ibidem, p. 197 : La ville de Rome [...] retourna à l'autorité consulaire grace à laquelle elle a soumis ses voisins et subjuguée l'Italie et elle porta sa puissance vers l'extérieur pour augmenter son Empire ; et la Monarchie castillane, [...] en chassant les Mahométans d'Espagne, poussa ses armées jusqu'en Italie et vers d'autres terres ».

27.Ibidem, p. 197 : «Qui l'a conduite à la très heureuse grandeur qui est la sienne à présent $»$.

28. Herrera, op. cit., p. 12 : «Et surtout qu'il ait l'esprit libre : celui-là seul fera bien son métier d'historien, ce qu'il ne pourra pas faire s'il a peur, ou s'il peut nourrir l'espoir d'un grand prix, ou s'il est mu par une haine quelconque ».

29. Antonio Domínguez Ortiz, « La censura de obras históricas en el siglo XVII español », Chronica Nova, Revista de Historia Moderna de la Universidad de Granada, n²19, 1991.

30. José Godoy Alcántara, Historia crítica de los Falsos Chronicones, Madrid, Ribadeneyra, 1868. Édition (facsimil) critique : Ofelia Rey Castelao (Estudio preliminar), op. cit., Granada, Editorial Universidad de Granada, 1999.

31. José Godoy Alcántara, Historia de los falsos cronicones, Madrid, 1868, p. 170.

32. Luís Cabrera de Córdoba, Historia de Felipe II, Rey de España, dir. Martínez Millán, Junta de Castilla y León, Consejería de Educación y cultura, 1998. Dans l'introduction, l'auteur justifie l'insertion du récit des événements d'Aragon et les notes marginales de Leonardo Argensola sur le même texte.

33. J.- F. Lazaro, El criterio historiográfico del Cronista Mayor D. Antonio de Herrera y Tordesillas (importancia del pleito del Conde de Puñoenrostro), Universidad de La Plata, p. 77-128, Buenos Aires, 1941.

34. cf. « Súplica del Conde Puñonrostro al Rey contra Herrera », 22 de mayo de 1603, Valladolid, A.G.I., Patronato, 18, citée par Mariano Cuesta Domingo.

35. «Información y razones por las que ha escrito y cómo lo ha hecho sobre Pedrarias Dávila », Ibidem.

36.Antonio de Herrera y Tordesillas, op. cit., p. 13.

37. Diálogos muy subtiles y notables, hechos por el illustrissimo y Reverendissimo señor Don Pedro de Navarra, Obispo de Comenge. Van dirigidos al muy Cathólico Rey de España don Phelippe nuestro Señor, impressos en Caragoça por Iuan Millan en la Cuchilleria, año de 1567. Voir en particulier Diálogos (I-V), ¿Quál deue ser el Chronista del principe?

Materia de pocos aun tocada. Pour une analyse de ces textes voir notre thèse de doctorat 
inédite : L'essor de la pensée historique en Espagne au Siècle d'Or, De Joan Luís Vives à Antonio de Herrera y Tordesillas, Université d'Aix en Provence, 2003.

38. Voir l'hommage de Diego José Dormer, Progressos de la historia en el reyno de Aragón y elogios de Gerónimo Zurita, su primer coronista... : ideó esta obra y la dispuso con las noticias que no tienen señal [...] Iuan Francisco Andrés de Uztarroz..., Zaragoza, por los Herederos de Diego Dormer, 1680.

39. Herrera, Discurso sobre que Tácito excede a todos los historiadores antiguos, y el fruto que se saca de sus escritos, op. cit., p. 44.

40. Selon les termes de Pierre Bourdieu.

41. Herrera, op. cit., p. 44 : « Les Crétois donnèrent une grande quantité d'argent à Homère pour ses œuvres. Nicomèdes, roi de Chypre, donna à Isocrates 120 ducats pour le livre qu'il lui dédia. Les Romains ont élevé une statut à Josèphe pour l'histoire qu'il rédigea sur la captivité des juifs."Et notre Roi Alphonse VIII nomma Rodrigue Jiménez de Rada primat des Espagnes, Archevêque de Tolède ».

42. Voir Georges Martin, Les juges de Castille. Mentalités et discours historique dans l'Espagne médiévale, Paris, Klincksieck (Annexes des Cahiers de linguistique hispanique médiévale, 6), 1992, p. 201-316.

43. Herrera, op. cit., p. 44 : « Nous n'aurions plus rien, si ce n'était grâce la grandeur d'âme et au dévouement qu'on éprouvé pour les belles lettres le Pape Léon X, qui les a sauvées des mains des barbares ».

44. Addition à l'histoire de Louis $\mathrm{XI}$, contenant plusieurs recherches curieuses sur diverses matières [Texte imprimé], par Gabriel Naudé... Paris, F. Targa, 1630.

45. « C'est une maxime avancée par les auteurs politiques et confirmée par une infinité d'exemples que les armes et les études ont coutume de fleurir en même temps, et que l'accroissement des Empires a été le plus souvent accompagné de celuy des lettres. Ainsi voyons-nous que le siècle de Philippe et Alexandre nous a donné Platon, Aristote et Démosthène ; celui d'Auguste, Varon, Virgile, Horace, Ovide, Strabon, Dioscoride, TiteLive, Saluste et Diodore [...] », ibidem, IV, p. 34-35, cité par Yves-Charles Zarka dans "L'idée d'une historiographie critique chez Gabriel Naudé », in Corpus, Revue de philosophie, $n^{\circ} 35$, sous la direction de Robert Damien et Yves-Charles Zarka, Centre d'Etudes d'Histoire de la Philosophie Moderne et Contemporaine, Université de Paris X, 1999, pp. 11-12.

46. Herrera, Primera parte de las varias Epístolas, Discursos y Tractados de Antonio de Herrera a diversos claros varones, las cuales contienen muchas materias utiles para el govierno político y milita. Con un elogio de la vida y hechos de el Lizdo. Xptoval Vaca de Castro del Consejo Supremo y Gobernador de los Reynos del Pirú, firma autógrafa del copista Fernando de Vallejo (fol. 189), BNM, Mss. 3011, fol. 105 : « Comme je me souviens d'avoir vu parmi les livres dont vous disposez quelque traité d'Annius de Viterbe signé Bérose, je vous adresse ce qu'après beaucoup d'étude j'ai pu trouver chez des auteurs fiables (des autorités) afin que vous sachiez combien d'hommes de valeur ont déjà été abusés et afin que personne d'autre, dans la mesure du possible, ne le soit encore ». 47. Ibidem : « [...] Il a commis de très grandes erreurs dans la chronologie [...] dont dépend la rigueur de l'histoire. Et ainsi, des hommes de grande valeur et de bonne religion, faute de percevoir le piège, sont tombés dedans, comme Jean Driedon, Nauclerus, Valère Anselme... ».

48. Melchor Cano, De locis theologicis libri duodecim, in Opera : Editio accuratior justa exemplar Salmanticae ex aisum, Lugduni \& veneunt Parisiis : apud. Amabilem Auroy, 1704. Nous avons consulté le Liber Undecimus, pp. 374-454 : « De Humanæ historiæ 
auctoritate quae postremo loco est posita. » (BNM, 3/17210.). La vérité ne doit pas s'écrire en langue romane : à cet égard il est intéressant de voir le théologien de la ContreRéforme reconnaître en latin ses dettes envers l'œuvre castillane de Juan de Vergara. Il avoue mettre largement à profit la réfutation des affabulations d'Annius de Viterbe dans ces lignes extraites de la page $418:$ : Atque eas omnes figillatim Joan Vergara canonicus Toletanus, vir Græcæ ac Latinæ linguæ peritissimus in libello 8 quæstio Hispane edito accuratius refutavit. Cujus no opera \& diligentia multum hoc loco adjuti sumus. ». 49. Tratado de las ocho qvestiones del templo propuestas por el Illmo. Señor Duqve del Infantadgo, respondidas por el doctor Vergara, Canónigo de Toledo, Toledo, 1552.

50. Antonio de Herrera y Tordesillas, op. cit., BNM, Mss. 3011, fols. 105-106 : « Nombreux sont les auteurs qui ont enquêté sur la vérité et l'ont mise en lumière, et en particulier frère Melchor Cano dans le livre 11 de ses Loci Comunes de théologie, Volterrano dans son livre 14 de l'Anthropologie, Juan de Vergara dans ses Ocho cuestiones del Templo, Luís Vives dans le livre V du De disciplinis ».

51. Ibidem : « Le véritable Bérose a écrit son histoire jusqu'à Cirus, qui vécut plus de 600 ans après la guerre de Troie et ce Bérose qu'invente Annius ne rapporte l'histoire des Assyriens et Chaldéens que jusqu'au début du royaume de Troie, c'est à dire jusqu'au dixhuitième roi des Assyriens, qui fut un contemporain de (Dardanos ?), le premier roi de Troie ».

52. Ibidem : « De surcroît, que l'on compare avec ce qu'a écrit Josèphe dans le Contra Apion : les livres du véritable Bérose étaient trois, et évoquait les Chaldéens alors que chez Annius il semble n'y en avoir qu'un, qui n'évoque que les Assyriens ».

53. Ibidem : « Annius lui-même contredit son Metasthene puisqu'il dit que Bérose écrivit l'histoire des rois d'Assyrie jusqu'à Sardanapale, dernier roi de cette monarchie, un mensonge évident et notoire ».

54. Ibidem : «Il est certain et bien connu que depuis Cyrus jusqu'à Alexandre le Grand il y a eu 14 rois de Perse y que (la dynastie) dura 230 années. Or ce Metasthenes qu'invente Annius ne présente que huit rois, et prétend que l'Empire des Perses n'a pas duré plus de 190 années ».

55. Ibidem : « Le $3^{\text {ème }}$ auteur qu'Annius a aussi utilisé à mauvais escient est Manethon, qui a écrit sur les Egyptiens très différemment du véritable ; le quatrième est Philon dont il prétend qu'il a écrit un bréviaire de l'histoire ».

56. Herrera, op. cit., p. 109 : « Rien ne semble propre à ce Philon d'Annius, le style est excellent, l'éloquence est la règle ainsi que la gravité, la fidélité et la vérité, dont on ne retrouve rien dans ce Philon ».

57. Herrera, op. cit., « Resumen de los que pasó en el descubrimiento de las santas reliquias de la ciudad de Granada, conforme a lo que escribió el Doctor Gregorio López. », BNM, Mss., 3011, fols. 183-191.

58. Herrera, op. cit., fol. $185 b$.

59. Julio Caro Baroja, Op. Cit., p. 133, signale deux traités selon les bibliographes aujourd'hui fort rares. Le premier s'intitule : Discurso sobre las láminas reliquias y libros que se an descubierto en la ciudad de Granada este año de 1595. Y las reliquias y prophecía que se avía hallado el año pasado de 1588. Del licenciado López Madera, fiscal de su magestad en la real chancillería de la dicha ciudad, Granada, Juan René, 1595, en folio con dos láminas. D'après Antonio Palau y Dulcet, Manual del librero hispanoamericano, Barcelona, 1926, T. IV, p. 267 a. Le second est la Historia y discurso de la certidumbre de las reliquias, láminas y prophecía descubiertas en el Monte Santo y iglesias de Granada desde 1580 hasta 1598, Granada, Sebastián de Mena, 1601 o 1602. 
60. Herrera, op. cit., fol. 183 : «En l'an très heureux de 1588 en détruisant une vieille tour dans la ville de Grenade, on trouva une boîte en plomb, scellée de l'intérieur et de l'extérieur ».

61. Ibidem, « Un récit en latin du prêtre Patrice, qui avance que l'écriture est une prophétie de Saint Jean l'Evangéliste et qui que la toile est la moitié de celle avec laquelle notre dame a essuyé ses larmes lors de la passion de son fils bénit ».

62. Ibidem, fol. 184a : «Une masse de cendres et d'os brûlés et une tête de mort, des dents et des molaires, le tout baignant dans une forte odeur et fragrance ».

63. Ibidem, fol. $184 \mathrm{~b}$.

64. Ibidem, fol. 185a : «Ces quatre lames et la couverture des livres annoncent un fundamentum ecclesia y essencia Dei et tout est écrit très habillement, comme par quelqu'un qui savait comment écrire sur du plomb, pour plus de pérennité ».

65. Ibidem, fol. 185b : «Les experts des divers arts ont tous déclaré officiellement qu'ils sont anciens de plus de mille ans, bien authentiques et sans artifice; et les Arabes savants que l'on fit venir de toutes parts dirent aussi que la grammaire était très ancienne ». 66. Ibidem, fol. 185b : « La grammaire, les phrases et le style, certaines formules et des lettres sans points, [sachant] que les points distinguaient en langue arabe des lettres plus ou moins appuyées, et qu'il aurait été impossible à des Arabes modernes de composer (ce texte) ; si cent Arabes avaient collaboré depuis cent ans à cette œuvre, ils n'en auraient pas été capables ».

67. Ibidem, fol. 187 : « Pour traiter un dossier aussi grave avec le tact et l'autorité de circonstance, l'Archevêque décida d'un jour où l'on procèderait à la qualification des reliques et il en informa le Nonce $»$.

68. Ibidem, fol. 187b : « Les conseils et les grands prélats de Castille et d'Andalousie, les abbées de toutes les provinces et les hauts dignitaires des ordres monastiques et mendiants, les professeurs des universités et les ministres du Saint Office de l'Inquisition ".

69. Ibidem : « En leur demandant instamment de rendre cet acte officiel par leur présence, comme l'exigeait l'importance de l'affaire, et afin qu'elle soit traitée conformément aux décrets de sa Sainteté et à la volonté du Roi et de son conseil, publiquement dans tout l'archevêché de Grenade ».

70. Ibidem, fol. 188a.

71. Cf. Nicolás Antonio, Bibliotheca Hispana Nova, I, pp. 545b-546a.

\section{RÉSUMÉS}

Diplomate, traducteur du tacitiste Giovanni Botero et secrétaire des grands serviteurs de la Monarchie hispanique, l'historien Antonio de Herrera y Tordesillas (1549-1625) a illustré une étape décisive de la conception et de l'écriture de l'histoire à l'époque de la Raison d'Etat. Cet historien castillan a magistralement formulé l'intérêt de la discipline, de sa connaissance et de son écriture, pour la pratique politique. En montrant que l'histoire est un miroir du prince selon la définition cicéronienne (historia magistra vitæe) à l'usage du souverain, il revendique plus que la dignité de sa fonction. 
S'il en appelle en effet à la constitution d'un champ savant des plus ambitieux, c'est parce qu'il estime que l'histoire peut mobiliser, autour de la Monarchie et des Castillans, l'ensemble des forces disponibles pour la mission hispanique universelle. Le récit des exploits castillans légitime un socle identitaire d'un nouvel ordre, fondé sur la durée, sur le temps de la formation d'une conscience partagée.

Enfin, si la définition des conditions de possibilité de l'émergence d'une vérité historique -un enjeu croissant des luttes idéologiques entre Etats modernes- est l'un des grands mérites de cet historien, Herrera n'a pas toujours su reconnaître les falsifications documentaires de son temps. Il illustre ainsi les limites moins spécifiquement méthodologiques que sociales de la critique historique de l'époque.

Diplomata, traductor del tacistista Giovanni Botero y secretario de los grandes servidores de la Monarquía hispánica, el historiador Antonio de Herrera y Tordesillas (1549-1625) ilustró una etapa decisiva de la concepción y de la escritura de la historia en la época de la Razón de Estado. Aquel historiador castellano formuló magistralemente el interés de la disciplina, de su conocimiento y de su escritura, para la práctica política. Mostrando que la historia es un espejo del príncipe según la definición ciceroniana (historia magistra vitæ) para uso del soberano, reivindica más que la dignidad de su función.

Si invoca la constitución de un campo científico de lo más ambicioso, es porque considera que la historia puede mobilizar, en torno a la Monarquía y los castellanos, el conjunto de las fuerza disponibles para la misión hispánica universal. El relato de las hazañas catellanas legitima un núcleo identitario de nuevo tipo, basado en la duración, en el tiempo histórico de la formación de una conciencia compartida.

Por fin, si la definición de las condiciones de posibilidad de la construcción de una verdad histórica -una dimensión creciente de las luchas entre estados modernos- es un mérito indudable del historiador, Herrera no pudo siempre denunciar las falsificaciones documentales de su tiempo. Ejemplifica también los límites no ya tanto metódicos sino más bien sociales de la crítica historica de la época.

\section{INDEX}

Mots-clés : histoire, falsification, faussaires, temps, Castille, Monarchie, Raison d'Etat, critique historique

Index chronologique : XVIIe siècle, XVIe siècle

\section{AUTEUR}

RENAUD MALAVIALLE

Université Paris IV, Sorbonne, CLEA, EA 2559 